\title{
EFFECT OF GAS DENSITY AND VISCOSITY ON THE MAXIMAL EXPIRATORY FLOW-VOLUME RELATIONSHIP
}

\author{
By DONALD P. SCHILDER,* ALBERT ROBERTS, † AND DONALD L. FRY
}

(From the Section of Clinical Biophysics, Cardiology Branch, National Heart Institute, Bethesda, Md.)

(Submitted for publication March 18, 1963; accepted July 18, 1963)

It has been shown that a functional relationship exists between transpulmonary pressure, respiratory gas flow, and the degree of lung inflation (1, 2). This relationship may be expressed in the form of a family of isovolumetric pressure-flow (PF) curves, each obtained at different degrees of lung inflation. Figure 1a shows four expiratory isovolumetric $\mathrm{PF}$ curves from a normal subject. The shape of each curve is different. Expiratory flows on PF curves 1, 2, and 3 were measured at volumes low in the vital capacity. Flow increases to the maxima $\mathrm{A}, \mathrm{B}$, and $\mathrm{C}$, and then diminishes with increasing pressure. On $\mathrm{PF}$ curves high in the vital capacity, such as curve 4 , effort adequate to achieve flow maxima cannot be developed. If the maximal flows from curves 1 through 4 are plotted against the volume at which each was measured, a maximal expiratory flow-volume (FV) curve results (Figure 1b). On the basis of the PF curves, this FV curve may be divided into two segments, the $\alpha-\beta$ segment and the $\beta-\gamma$ segment. The $\alpha-\beta$ segment (the solid portion of the curve in Figure 1b) extends over the lower portion of the vital capacity and corresponds to the $\mathrm{PF}$-curve maxima. The $\beta-\gamma$ segment (the dashed portion of the curve in Figure $1 \mathrm{~b})$ is the remaining part of the FV curve and extends over the upper portion of the vital capacity. The $\beta-\gamma$ segment corresponds to the ends of the isovolumetric $\mathrm{PF}$ curves not having maxima.

In practice, it is not necessary to obtain the $\mathrm{FV}$ curve from PF curves as outlined above unless careful identification of the $\beta$ point is desired. The FV curve may be simply and directly

\footnotetext{
* Present address: Pulmonary Laboratory, VA Hospital, West Haven, Conn.

$\dagger$ Present address: Heart Disease Control Program, Bureau of State Services, U. S. Public Health Service, Department of Health, Education, and Welfare, Washington, D.C.
}

obtained by plotting expiratory flow on the $y$ axis and volume on the $\mathrm{x}$ axis of some recording device, such as an oscilloscope. If the subject performs expiratory vital capacity maneuvers of sufficiently varying effort (from gentle to maximal effort), then the outer perimeter of the superimposed loops representing each effort will be very close to the maximal expiratory flow rate that the subject can develop at every point in his vital capacity, or the FV curve for that subject.

The FV curve meets the requirements of a practical clinical or mass survey test of ventilatory function (3-7). It is relatively simply and quickly obtained, causes no discomfort to the patient, and is reproducible. Furthermore, the $\alpha-\beta$ segment of the curve is determined entirely by the properties of the lung parenchyma, respired gas, and intrathoracic airways $(8,9)$. Consequently, the $\alpha-\beta$ segment should be uniquely sensitive to intrapulmonary disease processes, and so give valuable clinical information. The major obstacle in the clinical use of the FV curve has been related to the quantification, analysis, and interpretation of the curve. So far, several empirical approaches have been used.

First of all, since the FV curve in various disease states varies greatly from normal curves $(2,3,5,6)$, useful information may be had by simple inspection and comparison of curves, noting maximal flows at particular volumes, or by computing the time course of flow and volume from the curve. The curve has therefore been suggested as a useful method of presenting at a glance an integrated picture of all the information contained in the usual tests of ventilatory function $(3,5,6,9)$.

Another approach is that suggested by Dayman (3). Although he obtains a somewhat different type of FV curve from that described above, it has the same general shape and probably contains essentially the same information. He ana- 


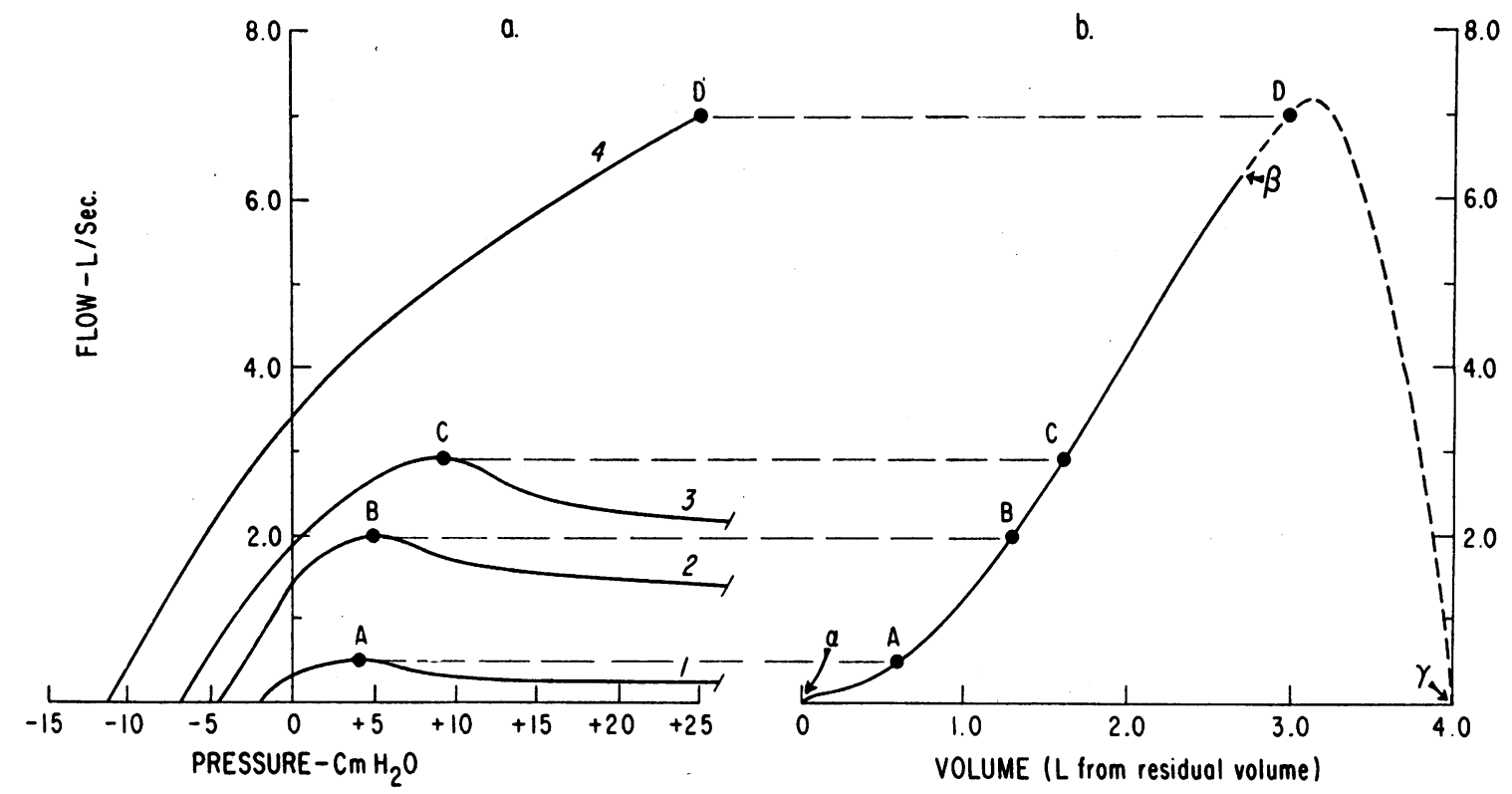

Fig. 1. a. A PLOT OF TRANSPULMONARY PRESSURE AND EXPIRATORY FLOW FOR A NORMAL SUBJECt AT DIFFERENT DEgREes of LUNG inflation. Curves $1,2,3$, and 4 were measured at $0.6,1.2,1.7$, and $3.0 \mathrm{~L}$, respectively, above residual volume. b. A PLOT OF MAXIMAL ACHIEVABLE EXPIRATORY FLOW AGAINST DEGREE OF LUNG INFLATION. Flow and volume coordinates of points A, B, C, and D from Figure la are plotted on this curve. The $\alpha-\beta$ segment of the flow-volume $(\mathrm{FV})$ curve corresponds to the maxima $(\mathrm{A}, \mathrm{B}$, and $\mathrm{C})$ on the isovolumetric pressureflow (PF) curves. Point $D$, the point of peak flow on isovolumetric curve 4, represents the maximal flow, but unlike $\mathrm{A}, \mathrm{B}$, and $\mathrm{C}$, is not a maximum, since flow is still increasing with pressure.

lyzes the FV curve by fitting the $\alpha-\beta$ segment with two straight-line segments, a long, steep line describing the major portion of the descending limb of the curve, and a shorter, less steep line describing the foot of the curve. He observed that the slope of the steeper portion of the curve was essentially constant for all normal subjects regardless of lung size. Subjects with obstructive disease, or increased lung compliance, or both, showed a marked decrease in this slope. Subjects with decreased compliance showed an increased slope. The slope of the foot of the curve in both health and disease was less consistent. Dayman therefore suggested that the most useful measurement to be taken from the curve is the slope of the steep portion. Moreover, the correlation of these slope measurements with compliance measurements has led him to postulate that lung compliance is one of the major determinants of the FV curve. Others have reached generally similar conclusions $(2,6,8-10)$.

Curves obtained by our methods do not clearly show the two linear components described by Dayman, so, originally, an empirical exponential function was fitted to the FV curve and tentatively used as a method of quantifying it (2). Subsequent experience has indicated that the curve deviates from the assumed function enough that this approach requires more art than science and is thus unsatisfactory.

Burger (6) has found empirically that the curve is best expressed as a quadratic relationship between volume and flow, i.e., flow is proportional to the square of volume. For clinical purposes, however, his group has found it most useful simply to characterize the curve by flow values measured at various specified points in the vital capacity. Burger emphasizes the value of these measurements in the early diagnosis of emphysema.

The most fruitful interpretation of the $\mathrm{FV}$ curve would be one establishing a correspondence between measurements from the curve and the physiological properties of the pulmonary system, but such an approach is possible only if one has a realistic mathematical model of the lung. Since the lung is a very complicated mechanical system, the search for a realistic model is a formidable task. 
The first step in attacking such a problem is to determine the gross behavior of the simplest model that is consistent with the major anatomical features as well as with the known pressureflow-volume relationships of the human lung. One such model has been analyzed theoretically (8). Inferences drawn from this analysis have led to the conclusion that the $\alpha-\beta$ segment of the FV curve must be determined exclusively by intrinsic pulmonary factors, i.e., the physical properties of certain portions of the lower airways as well as the density and viscosity of the expired gas, whereas the $\beta-\gamma$ segment of the FV curve is determined by the relationship of the total load presented by the respiratory system to laws governing muscular motor function.

The present study was designed to explore further the role of various factors intrinsic to the lung in determination of the $\alpha-\beta$ segment of the $\mathrm{FV}$ curve and the role of other factors in deter- mination of the $\beta-\gamma$ segment. Known changes were produced in the physical properties of the gas breathed in four normal subjects, and the effects on the $\alpha-\beta$ and $\beta-\gamma$ segments were observed. Changes in the $\alpha-\beta$ segment were compared with changes predicted from the behavior of a simple lung model; changes in the $\beta-\gamma$ segment were compared with changes predicted from theory of muscle behavior.

\section{METHODS}

FV curves were obtained repeatedly over 3 weeks on four trained, normal subjects while they expired gases of predetermined density and viscosity. The composition and physical properties of these are listed in Tab'e I. Curves obtained with the subject expiring normal expired gas (gas C) were considered control curves. Curves were also obtained while the subject expired a gas with the same density as gas $C$ but with a $44 \%$ greater viscosity (gas V), and while he expired a gas with approximately the same viscosity as gas $\mathrm{C}$ but with a $44 \%$ greater density (gas $D_{1}$ ). The $44 \%$ difference in

TABLE I

Physical properties of the various gas mixtures used

\begin{tabular}{|c|c|c|c|c|c|c|c|}
\hline , Gas & $\begin{array}{c}\text { Gas } \\
\text { components }\end{array}$ & $\begin{array}{l}\text { Composition } \\
\text { of inspired } \\
\text { gas }\end{array}$ & $\begin{array}{l}\text { Composition } \\
\text { of resulting } \\
\text { expired gas }\end{array}$ & $\begin{array}{l}\text { Density of } \\
\text { expired gas }\end{array}$ & $\begin{array}{l}\text { Viscosity } \\
\text { of expired } \\
\text { gas }\end{array}$ & $\begin{array}{l}\text { Density of } \\
\text { expired gas/ } \\
\text { density } \\
\text { of expired } \\
\text { gas C }\end{array}$ & $\begin{array}{l}\text { Viscosity of } \\
\text { expired gas/ } \\
\text { viscosity } \\
\text { of expired } \\
\text { gas C }\end{array}$ \\
\hline & & $\%$ & $\%$ & $\begin{array}{l}\mathrm{g} / \mathrm{L}, 37^{\circ} \mathrm{C} \\
750 \mathrm{~mm} \mathrm{H}\end{array}$ & upoise & & \\
\hline C, control & $\begin{array}{l}\mathrm{O}_{2} \\
\mathrm{CO}_{2} \\
\mathrm{H}_{2} \mathrm{O} \\
\mathrm{N}_{2}\end{array}$ & $\begin{array}{l}20.9 \\
79.1\end{array}$ & $\begin{array}{r}14.7 \\
4.9 \\
6.3 \\
74.1\end{array}$ & 1.08 & 180 & & \\
\hline $\mathrm{V}$, viscous & $\begin{array}{l}\mathrm{O}_{2} \\
\mathrm{CO}_{2} \\
\mathrm{H}_{2} \mathrm{O} \\
\mathrm{Ne} \\
\mathrm{SF}_{6}\end{array}$ & $\begin{array}{r}20.9 \\
\\
74.2 \\
4.9\end{array}$ & $\begin{array}{r}14.7 \\
4.9 \\
6.3 \\
69.5 \\
4.6\end{array}$ & 1.08 & 259 & 1.0 & 1.44 \\
\hline $\mathrm{D}_{1}$, dense & $\begin{array}{l}\mathrm{O}_{2} \\
\mathrm{CO}_{2} \\
\mathrm{H}_{2} \mathrm{O} \\
\mathrm{N}_{2} \\
\mathrm{SF}_{6}\end{array}$ & $\begin{array}{l}20.9 \\
\\
68.0 \\
11.1\end{array}$ & $\begin{array}{r}14.7 \\
4.9 \\
6.3 \\
63.7 \\
10.4\end{array}$ & 1.56 & 181 & 1.44 & 1.01 \\
\hline $\mathrm{D}_{2}$, more c'ense & $\begin{array}{l}\mathrm{O}_{2} \\
\mathrm{CO}_{2} \\
\mathrm{H}_{2} \mathrm{O} \\
\mathrm{N}_{2} \\
\mathrm{SF}_{6}\end{array}$ & $\begin{array}{l}20.9 \\
\\
32.6 \\
46.5\end{array}$ & $\begin{array}{r}14.7 \\
4.9 \\
6.3 \\
30.5 \\
43.6\end{array}$ & 3.11 & 166 & 2.88 & 0.92 \\
\hline $\mathrm{D}_{3}$, most dense & $\begin{array}{l}\mathrm{O}_{2} \\
\mathrm{CO}_{2} \\
\mathrm{H}_{2} \mathrm{O} \\
\mathrm{SF}_{6}\end{array}$ & $\begin{array}{r}20.9 \\
79.1\end{array}$ & $\begin{array}{r}14.7 \\
4.9 \\
6.3 \\
74.1\end{array}$ & 4.53 & 158 & 4.19 & 0.88 \\
\hline L, light & $\begin{array}{l}\mathrm{O}_{2} \\
\mathrm{CO}_{2} \\
\mathrm{H}_{2} \mathrm{O} \\
\mathrm{He}\end{array}$ & $\begin{array}{l}20.0 \\
80.0\end{array}$ & $\begin{array}{r}13.8 \\
4.9 \\
6.3 \\
75.0\end{array}$ & 0.375 & 202 & 0.35 & 1.12 \\
\hline
\end{tabular}


density or viscosity (with the other factor held constant) is the maximal difference that can be achieved unless one uses hydrogen, anesthetic gases, less than $21 \% \mathrm{O}_{2}$ in the inspired gas mixtures, or a pressurized chamber for the entire study. For a variety of reasons, none of these techniques were chosen. Expired gas density can be altered considerably, however, if viscosity is allowed to vary. In order to study the effect of larger changes in gas density, curves were also obtained with the subjects expiring gases much lighter or heavier than gas $C$, i.e., gases $L, D_{2}$, and $D_{3}$ described in Table $I$.

Volume and flow were measured with a Med-Science Electronics model 350 Servo-Spirometer. The calibration of this flow-measuring system is independent of the gas breathed. The dynamic response of this spirometer was found adequate to measure all but the initial rapid rise on the FV curve (initial part of dotted segment near $\gamma$ point as shown in Figure $1 \mathrm{~b}$ ). The peak flow value and the descending limb of the FV curve were found to be accurately recorded. Flow was plotted on the $\mathrm{y}$ axis and volume on the $\mathrm{x}$ axis of a Sanborn model $670 \mathrm{~A} x-y$ recorder having a uniform response $\pm 5 \%$ through 50 cycles per second.

A Tissot gasometer was filled with the gas mixture to be studied. The compositions of these gases appear in the "inspired gas" column of Table I. The density and viscosity that influence the $\mathrm{FV}$ curve are those of the expired gas, and are shown in the "expired gas" column of Table I. The subject's ventilating system was rinsed with the test gas from the gasometer before the FV curves were taken. Control curves were obtained after an identical rinsing procedure from a gasometer filled with room air. Toward completion of the rinse, the subject inspired maximally, and his respiratory stream was switched into the Servo-Spirometer so that the first forced expiratory vital-capacity effort could be measured. At the completion of this vital capacity effort, his respiratory stream was abruptly turned back to the rinse gasometer, from which he breathed quietly to avoid hypocapnia. The subject again inspired maximally from the rinse gasometer, and then his respiratory stream was turned into the Servo-Spirometer for another expiratory effort. This procedure was repeated 7 to 9 times for each gas mixture. The subject was carefully trained and coached to exert varying efforts with each expiration so that the maximal perimeter or envelope of the flow-against-volume diagram could be obtained. Three such envelopes or "FV curves" were obtained with gases $C, V, D_{1}$, and $D_{2}$ in every subject, with gas $L$ in three subjects, and with gas $D_{3}$ in a single subject. The three curves were averaged, and a mean curve was constructed for each gas in every subject.

In spite of the effort dependence of the $\beta-\gamma$ segment of the FV curve, the subjects were observed to achieve approximately the same peak flow. with a given gas when instructed to give repeated maximal expiratory efforts. This consistent response suggests that maximal neuromuscular effort, the load, and the flow are not independent. Therefore, isovolumetric PF curves from a single subject were also measured while he breathed gases $C, L$, and $D_{2}$, to examine maximal achievable pressure as a function of flow and volume. The intrathoracic pressure in this subject was estimated with an intraesophageal pressure-measuring system háving adequate recording characteristics.

\section{RESULTS}

The $\alpha-\beta$ segment. The results of this study indicate that variation in the viscosity and density of expired gas in these subjects caused unique and consistent changes in the FV curve. Three mean FV curves from one subject breathing gases $\mathrm{C}, \mathrm{V}$, and $D_{1}$ appear in Figure 2. Gas viscosity was found to affect the FV curve significantly only over the lowermost portion of the $\alpha-\beta$ segment. Increased viscosity caused a diminished flow and decreased slope of the curve in this region. To quantify this difference, the average slope for the gas $C$ curve was compared with the average slopes of the curves obtained by expiring gas $V$ and $D_{1}$. Since the lower part of the $\alpha-\beta$ segment is curvilinear, the average slopes were taken as the slope of the respective chords drawn from zero volume to the flow at $15 \%$ of the vital capacity (arrow 1

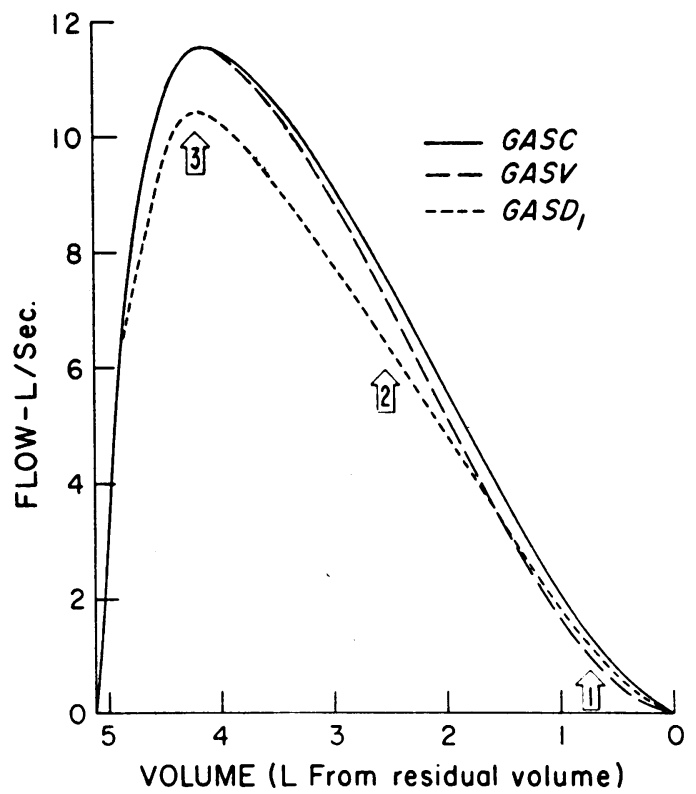

Fig. 2. Mean FV curves obtained with gases C, V, AND $D_{1}$ IN A single subject. Numbered arrows indicate regions of curve where effects of gas density and viscosity are compared and discussed. Arrow 1, lowermost portion or "foot" of $\alpha-\beta$ segment. Arrow 2, region of steepest slope of the $\alpha-\beta$ segment. Arrow 3, point of peak expiratory flow, in the $\beta-\gamma$ segment. 


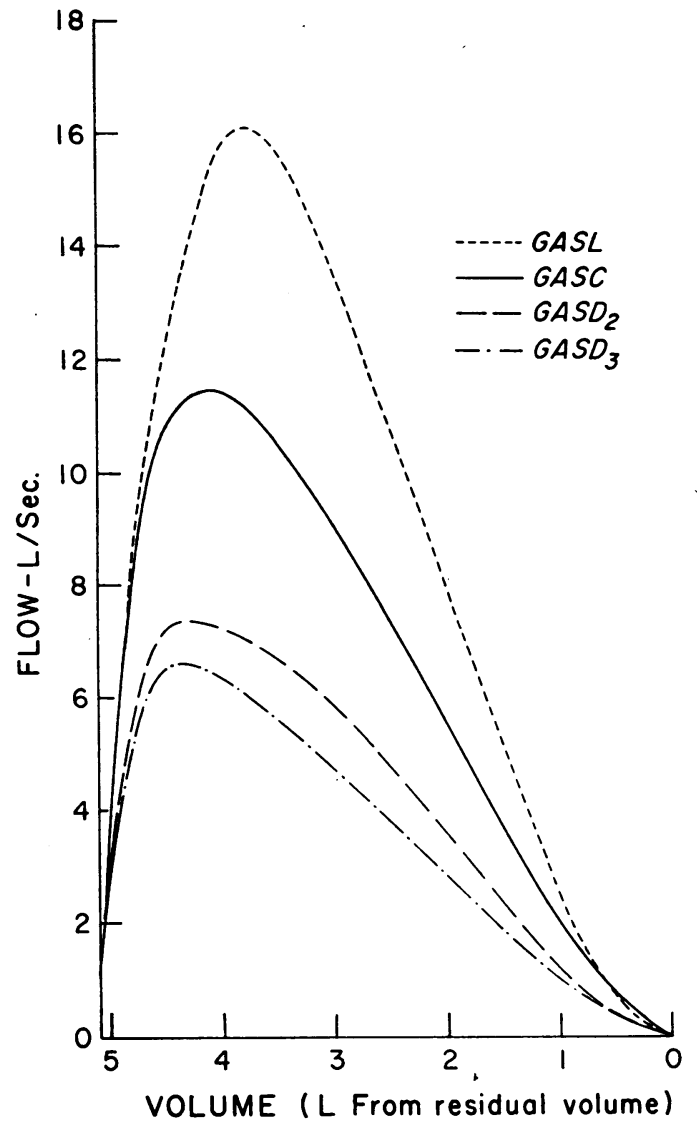

Fig. 3. Mean FV curves obtained with gases L, C, $\mathrm{D}_{2}$, AND $\mathrm{D}_{3}$ IN A SINGLE SCBJECT.

in Figure 2). Gas $\mathrm{D}_{1}$ (with a $44 \%$ increase in gas density) caused a $9.7 \pm 3.5 \%$ mean decrease in slope, whereas gas $\mathrm{V}$ (with a $44 \%$ increase in gas viscosity) caused a $23.6 \pm 4.2 \%$ mean decrease in slope. ${ }^{1}$ The average slope at the foot of the FV curve is thus significantly more depressed by an increase in viscosity than it is by an equal increase in density.

The upper part of the $\alpha-\beta$ segment is much more linear than the lower portion, so the effect of varying viscosity and density on this portion of the curve can be studied with a method similar to Dayman's by measuring the tangent or true slope of the curve in this region (arrow 2 in Figure 2). A $44 \%$ increase in gas viscosity did not alter the slope of the upper part of the $\alpha-\beta$ segment sig-

1 Expressed as mean $\pm \mathrm{SE} \times t$ with 3 degrees of freedom and a probability of .975 . This specifies a $95 \%$ confidence interval on the true percentage difference from control values. nificantly, whereas a $44 \%$ increase in gas density caused a significant change in slope. To explore this difference further, curves obtained with additional gases of widely varying densities were studied (gases $\mathrm{D}_{2}, \mathrm{D}_{3}$, and $\mathrm{L}$ ). Curves obtained in the same subject with these gases appear in Figure 3. The effect of gas density was quantified by the ratio of the steepest slope on the gas- $\mathrm{C}$ curve to the maximal slope of the respective testgas curves. These slope ratios were plotted against the ratios of the respective gas densities in Figure 4. The slope of the upper part of the $\alpha-\beta$ segment varies inversely with the density of the gas breathed. Moreover, this linear, logarithmic plot suggests that the relationship between slope and density could be expressed empirically by an equation of the form

$$
\frac{\text { slope } 1}{\text { slope } 2}=k\left(\frac{\text { density } 1}{\text { density } 2}\right)^{-n} \text {, }
$$

where $n$ is about +0.4 .

It is apparent, then, that the slope of the lower part of the $\alpha-\beta$ segment is most strongly influenced by gas viscosity, whereas the slope of the upper part of the $\alpha-\beta$ segment is most strongly influenced by gas density.

The $\beta-\gamma$ segment. The $\beta-\gamma$ segment of the FV curve was affected predominantly by gas density. A $44 \%$ increase in gas density produced a significant mean percentage decrease of $10.6 \pm$ $6.6 \%{ }^{1}$ in the peak expiratory flow achieved (arrow 3 in Figure 2). On the other hand, a 44\% increase in gas viscosity produced no significant change in peak expiratory flow. With all gases used, peak flow was decreased with increased

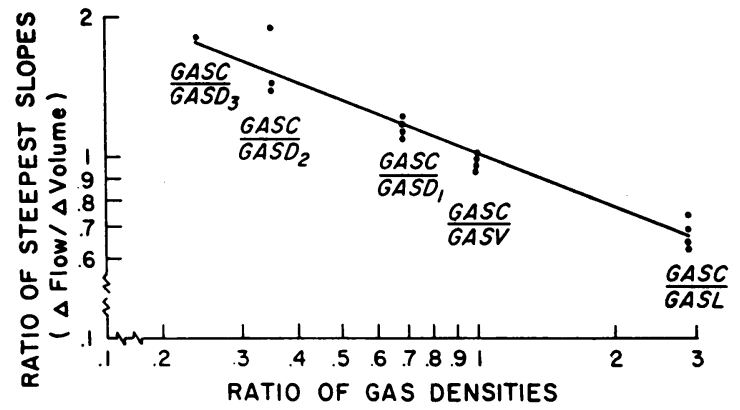

Fig. 4. Ratio of the steepest SLOPE oN the FV CURVE OBTAINED WITH GAS C TO THAT OBTAINED WITH THE OTHER GASES PLOTTED AGAINST RATIO OF THE DENSITY OF GAS C TO THE OTHER GASES. 
gas density as shown by the curves in Figure 3 for a single subject. The average decrease in peak flow for all subjects was $31 \%$ for gas $\mathrm{D}_{2}(188 \%$ more dense than gas $C$ ) and $47 \%$ for gas $\mathrm{D}_{3}$ ( $319 \%$ more dense than gas $\mathrm{C})$. With gas $\mathrm{L}$ (only $35 \%$ of the density of gas C), a mean increase of $46.1 \%$ in expiratory flow was achieved. Thus, gas density strongly influences the $\beta-\gamma$ segment of FV curve.

Maximal isovolumetric pressure-flow relationship. Three isovolumetric PF curves measured at a lung inflation of $3,800 \mathrm{~cm}^{3}$ from the residual volume while one subject breathed three different gases $\left(\mathrm{L}, \mathrm{C}\right.$, and $\mathrm{D}_{2}$ ) appear in Figure 5 . Circled points represent the maximal pressure and flow that could be achieved with each gas. The circled point on the abscissa is the maximal static pressure that the subject could exert. The dashed curve represents the maximal pressureflow relationship for this subject at a lung inflation of $3,800 \mathrm{ml}$.

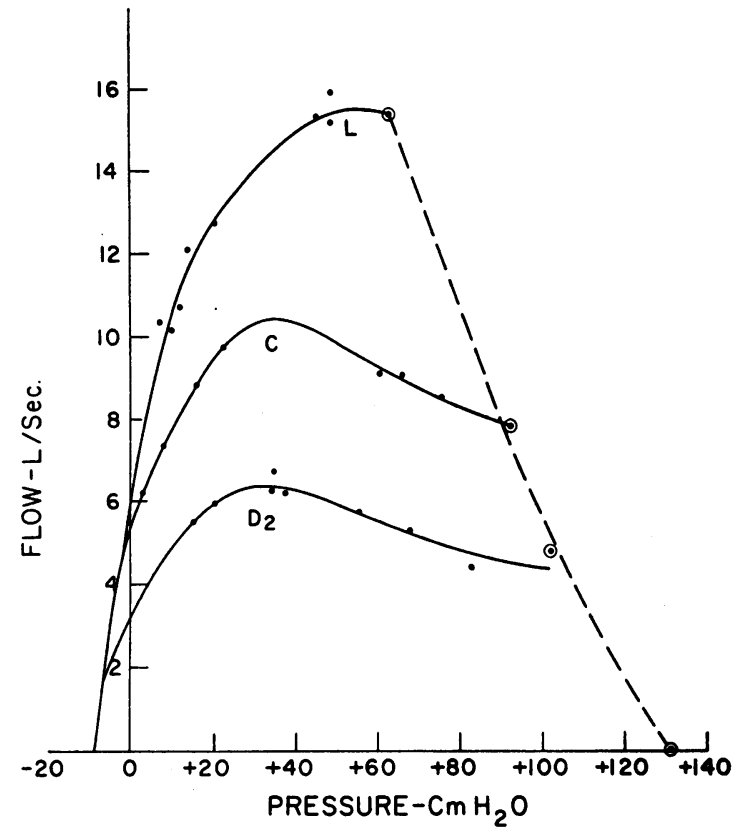

Fig. 5. Isovolumetric PF CURves obtained With Gases L, C, AND $D_{2}$ IN A Single SUbJect at a LUNG INFLATION 3,800 ML ABOve the RESidual volume. The zero flow-pressure point was obtained during a forced expiratory effort against a closed valve at the same lung volume. Circled points represent maximal intrathoracic pressures developed at this lung volume and gas flow. The dashed line drawn through these points to the zero flow point is the isovolumetric maximal pressure-flow relationship.

\section{DISCUSSION}

The $\alpha-\beta$ segment. As mentioned earlier, it would be most useful to analyze the FV curve in terms of the physiologic properties of the lung that determine its shape. Certain theoretical explorations to this end have led to the concept of a "flow-limiting segment" (8). With the lung assumed to be symmetrical and to have an even ventilatory distribution, the shape of the $\alpha-\beta$ portion of the FV curve was shown to be uniquely determined by the physical properties of the lung parenchyma, the flow-limiting segment, and that portion of the conduit system between this segment and the alveoli. Moreover, the curve was shown to be independent of all events downstream from the flow-limiting segment.

A tutorial discussion of the salient features of the theory using a grossly simplified model has also been presented (10). It is instructive to compare the results of our study and other published observations with the predicted behavior of this simple model. In it, that portion of the lung extending from alveolus to the flow-limiting segment was represented by a chamber (chest cavity) containing a single elastic air space (lung parenchyma) ventilating to the atmosphere through an elastic airway (bronchial system from alveolus to flow-limiting segment). The model predicts that the maximal expiratory flow $(Q)$ that can be achieved at any degree of lung inflation $(V)$ is given by

$$
Q=\frac{1}{2 K_{1}}\left[\frac{R_{0}^{2}}{2 K_{2}}+\frac{R_{0} V}{C}+\frac{{ }^{\prime} K_{2} V^{2}}{2 C^{2}}\right],
$$

where $K_{1}$ is a flow "resistance coefficient" of the intrathoracic airways, $R_{0}$ is the resting radius of the elastic tube, $K_{2}$ is a circumferential rigidity coefficient for the tube, and $C$ is the compliance of the elastic airspace. Inspection of Equation 2 shows that flow is a quadratic function of volume and thus represents a curve that will resemble the experimental $\alpha-\beta$ portion of the FV curve as found by Burger (6). The slope of the $\alpha-\beta$ segment of the model's FV curve will be given by the derivative of Equation 2:

$$
\text { slope }=\frac{d Q}{d V}=\frac{1}{K_{1} C}\left[\frac{R_{0}}{2}+\frac{K_{2} V}{2 C}\right] .
$$

The slope of the curve at any point will thus be proportional to $1 / K_{1} C$, where $K_{1} C$ is the product 
of lung compliance times the flow "resistance coefficient." By electrical analogy, this product may be called a "time constant." Therefore, the slope of the $\alpha-\beta$ portion of the FV curve will vary inversely with the "time constant" of the lung from the flow-limiting segment peripherally. Certain published data are interesting for correlation of this prediction with previous observations.

As mentioned earlier, Dayman (3) and also Branscomb (4) have shown that the slope of the FV curve is relatively constant from person to person in normal subjects regardless of their size. Interpreted by the model above, this constancy would suggest that the $K_{1} C$ product was independent of body size. Frank, Mead, Siebens, and Storey (11) have shown a positive correlation of lung compliance $(C)$ with height, body surface area, and observed vital capacity. Briscoe and DuBois (12) have showed a positive correlation of the total airway conductance (the reciprocal of airway resistance) with lung volume. Since lung volume and body size are correlated, lung conductance should show a positive correlation with body size. Although these conductance measurements were made at relatively low flows and for the airway as a whole, it is reasonable to assume that portions of the airway between corresponding points in different persons might also show a positive correlation between conductance and body size. To the extent, then, that the flowlimiting segment is the same from person to person in normal subjects, the fraction $1 / K_{1}$ should also show a positive correlation with body size, or conversely, $K_{1}$ should vary inversely with size. It follows that $C$, which is about proportional to size, $\times K_{1}$, which is inversely proportional to size, should become relatively independent of size, i.e., $K_{1} C$ should be constant from person to person in normal subjects. This inference, drawn from considerations of airway resistance and lung compliance measurements, is consistent with the observation that the slope of the FV curve is also independent of body size if these observations are interpreted with the predictions of the model (Equation 3). These considerations support Dayman's original contention that the slope of the FV curve appears empirically to contain the most useful clinical information and that lung compliance appears to be an important determinant of the FV curve.
Now let us see how the model fits our findings. Consider the flow "resistance coefficient" $K_{1}$. In the original derivation of Equation 2, $K_{1}$ was greatly simplified for tutorial purposes. As discussed elsewhere, this simple "constant" should, in fact, be replaced by a series of equations representing the viscous effects, turbulent effects, the Bernoulli effect, and all other complex pressureflow relationships occurring in elastic tubes (8, 9 ), but a hopelessly complicated set of equations would result. Therefore, as a first approximation for the sake of simplicity, $K_{1}$ may be replaced by a "flow resistance" expression modified from Rohrer (13),

$$
K_{1}=\left(k_{1} \mu+k_{2} \rho Q\right),
$$

where $k_{1}$ and $k_{2}$ are constants determined from certain geometric considerations of the airway, $Q$ is the flow, and $\mu$ and $\rho$ are the viscosity and density of the expired gas. In Equation 4, the pressure drop along the tube is assumed to be the sum of a linear Poiseuille-type pressure drop plus a quadratic density-dependent pressure drop related to energy loss in eddy formation. Also, bronchial compression during expiration is assumed to affect both terms proportionately. Although the effect of true turbulence and its distribution along the tube is ignored, this constitutes only a minor objection, since these turbulent effects are probably only important in the airway above the flow-limiting segment, and so would have no influence on the FV relationships (8). Thus, the flow resistance as usually defined, namely, as the ratio of the pressure drop along a given airway to the corresponding flow, becomes a function of gas viscosity, gas density, the flow, and certain geometrical properties. With $K_{1}$ replaced in the derivation of Equation 2, the slope of the $\alpha-\beta$ segment at a given volume in a given person can be shown to vary inversely with $k_{1} \mu+2 k_{2} \rho Q$. Therefore, the ratio of the slope of the $\alpha-\beta$ segment when the subject is breathing a gas with a viscosity $\mu_{1}$ and density $\rho_{1}$ to the slope at the same volume when he is breathing a gas of viscosity $\mu_{2}$ and density $\rho_{2}$ would be given approximately by

$$
\frac{S_{1}}{S_{2}}=\frac{k_{1} \mu_{2}+2 k_{2} \rho_{2} Q}{k_{1} \mu_{1}+2 k_{2} \rho_{1} Q} .
$$

Note that as flow approaches zero, this ratio ap- 
proaches the reciprocal of the viscosity ratios, and density becomes unimportant. Thus, the slope will be most sensitive to viscosity changes at low flows, or at the foot of the model's $\alpha-\beta$ segment. As was shown above, the $\alpha-\beta$ segments obtained during expiration of gas $\mathrm{V}$ (more viscous) had a significantly lower average slope than gas $\mathrm{C}$ or gas $\mathrm{D}$ at the foot of the curve. As a matter of fact, even gas- $\mathrm{L}$ curves (much less dense but $12 \%$ more viscous) dipped below the control curves in every subject (Figure 3). The model's prediction for the effect of viscosity agrees qualitatively with the observed data.

The limit of Equation 5 at very high flows is controlled by the ratios of the densities and becomes relatively insensitive to viscosity. Thus, the steepest slope of the model's $\alpha-\beta$ segment appears to vary approximately inversely with the gas density. The steepest slopes of the experimental curves, shown in Figure 4, varied inversely with the gas density. Once again, the prediction from the simple model agrees qualitatively with our data from studies in man.

Maximal isovolumetric pressure-flow relationship. As discussed earlier, the $\beta-\gamma$ segment of the FV curve depends on the maximal muscular effect the subject can exert. Since small changes in effort greatly alter the upper portions of these curves, it is interesting that subjects instructed to perform with maximal effort respond with surprising consistency. This suggests that the respiratory neuromuscular system responds to a command from the central nervous system for "maximal effort" in a way perhaps analogous to a maximally stimulated muscle. Hill (14) has shown that a maximally stimulated skeletal muscle shortens with a velocity that varies inversely with the load that the muscle must overcome. Agostoni and Fenn (15) have suggested that the classical force-velocity relationship might be applied to the respiratory musculature during maneuvers of maximal effort. They were able to demonstrate an inverse relationship between an estimate of mean inspiratory flow and mean airway pressure over the mid-range of the vital capacity during respiratory efforts against varying airway obstructions.

Our study shows in Figure 5 that maximal flow varies inversely with pressure in a manner consistent with but not necessarily indicating an underlying classical force-velocity relationship. The dashed line drawn through the maximal pressure points that can be achieved at that particular lung volume and flow could be called an isovolumetric maximal $\mathrm{PF}$ relationship. Although for present purposes the maximal PF curve was obtained by variation of intrathoracic airway resistance, it could have been obtained just as well by variation of extrathoracic airway resistance.

The significance of this maximal PF curve is that it represents the limit of effort that is available for ventilation in a given subject. A subject's ability to sense the relationship between this limit and the magnitude of the imposed ventilatory load suggests one potentially fruitful approach to the complex problem of dyspnea.

\section{SUMMARY}

The maximal expiratory flow that can be developed throughout the vital capacity is called the flow-volume (FV) curve. This curve is divided into the $\alpha-\beta$ and the $\beta-\gamma$ segments. The $\alpha-\beta$ segment spans the lower two-thirds of the vital capacity and corresponds to the maxima of the isovolumetric pressure-flow (PF) curves in this region. The $\beta-\gamma$ segment spans the remainder of the vital capacity and corresponds simply to the maximal effort that can be developed. The $\alpha-\beta$ segment of the FV curve is determined entirely by the properties of the lung, respired gas, and intrathoracic airway. The $\beta-\gamma$ segment of the FV curve is determined by the mechanical impedance of the over-all pulmonary system and the laws governing motor function. Variation of the viscosity and density of expired gas caused unique and consistent changes in the FV curve. The lower part of the $\alpha-\beta$ segment is most strongly influenced by gas viscosity, whereas the upper part is most strongly influenced by gas density. The results agree qualitatively with theoretical predictions based on a simple lung model. This model also predicts that the slope of the $\alpha-\beta$ segment of the FV curve would vary inversely with the product of lung compliance and a lower airway "resistance constant." Published evidence supports this hypothesis. These considerations suggest that the slope of the FV curve should be a clinically useful index relating lung compliance to airway resistance. 


\section{ACKNOWLEDGMENT}

The authors gratefully acknowledge the statistical assistance of Mr. Robert H. Baird of the Biometrics Research Branch, National Heart Institute.

\section{REFERENCES}

1. Fry, D. L., R. V. Ebert, W. W. Stead, and C. C. Brown. The mechanics of pulmonary ventilation in normal subjects and in patients with emphysema. Amer. J. Med. 1954, 16, 80.

2. Hyatt, R. E., D. P. Schilder, and D. L. Fry. Relationship between maximum expiratory flow and degree of lung inflation. J. appl. Physiol. 1958, 13, 331.

3. Dayman, H. The expiratory spirogram. Amer. Rev. resp. Dis. $1961,83,842$.

4. Branscomb, B. The application of the respiratory flow-volume loop in epidemiologic surveys. Amer. Rev. resp. Dis. 1962, 86, 697.

5. Hyatt, R. E. The interrelationships of pressure, flow, and volume during various respiratory maneuvers in normal and emphysematous subjects. Amer. Rev. resp. Dis. 1961, 83, 676.

6. Burger, $H$. C. The significance of the flow/volume diagram in the study of the mechanics of breathing. Proc. Tuberc. Res. Counc. 1959, 46, 28.

7. Bartlett, R. G., Jr. Pulmonary function evaluation in air and space flight. Aerospace Med. 1961, 32, 685 .
8. Fry, D. L. Theoretical considerations of the bronchial pressure-flow-volume relationships with particular reference to the maximum expiratory flow volume curve. Phys. in Med. Biol. 1958, 3, 174.

9. Fry, D. L., and R. E. Hyatt. Pulmonary mechanics. A unified analysis of the relationship between pressure, volume and gas flow in the lungs of normal and diseased human subjects. Amer. J. Med. 1960, 29, 672.

10. Fry, D. L. The physics of air flow in emphysema. Amer. Rev. resp. Dis. 1959, 80, 123.

11. Frank, N. R., J. Mead, A. A. Siebens, and C. F. Storey. Measurements of pulmonary compliance in seventy healthy young adults. J. appl. Physiol. 1956, 9, 38.

12. Briscoe, W. A., and A. B. DuBois. The relationship between airway resistance, airway conductance and lung volume in subjects of different age and body size. J. clin. Invest. 1958, 37, 1279.

13. Rohrer, F. Der Stromungswiderstand in den menschlichen Atemwegen und der Einfluss der unregelmassigen Verzweigung des Bronchialsystems auf den Atmungsverlauf in verschiedenen Lungenbezirken. Arch. ges. Physiol. 1915, 162, 225.

14. Hill, A. V. The heat of shortening and the dynamic constants of muscle. Proc. roy. Soc. B 1938, 126, 136

15. Agostoni, E., and W. O. Fenn. Velocity of muscle shortening as a limiting factor in respiratory air flow. J. appl. Physiol. 1960, 15, 349. 